Polymer Journal, Vol. 6, No. 6, pp 455-460 (1974)

\title{
Angular Relations between Two Polypeptides in Hinged Polymers
}

\author{
Hiroshi Kinara* and Akiyoshi WadA \\ Department of Physics, Faculty of Science, Tokyo University, \\ Bunkyo-ku, Tokyo 113, Japan. \\ (Received October 24, 1973)
}

\begin{abstract}
To investigate the flexibility of the coiling part of hinged polymers (two $\alpha$-helices joined by a coil), the energy contour map which represents the allowed region in the configurational space of the hinge has been constructed. It can be concluded from the maps that the hinge is perfectly or nearly perfectly flexible at room temperature.
\end{abstract}

KEY WORDS Hinged Polymer / Poly( $\gamma$-benzyl glutamate) / Energy Contour Map /

A hinged polymer which is composed of two $\alpha$-helices joined by the coiling part of the bifunctional initiator used in the polymerization can be considered a suitable model for investigating the interaction between two closely approaching $\alpha$-helices. ${ }^{1-3}$

Measurements of the dielectric dispersion of such polymers in dilute solution ${ }^{1}$ show that the average angle between the two helices, $\Theta$, is a little larger than $90^{\circ}$, which can be explained by considering the electrostatic interaction between the two helices. To make sure whether the electrostatic interaction alone is involved or whether the rigidity of the hinge has some effect on this angle, the internal energy at the hinge has been examined in this report. The energy contour map which represents the allowed region in the configurational space of the hinge has been made, where only the interactions between nearest neighboring groups have been considered. It has been confirmed from molecular model building that the long-range steric hindrance does not change the conclusion mentioned above.

\section{CALCULATIONS AND RESULTS}

The structural formula of the initiator with two glutamate residues at each end is shown in Figure 1, where the atoms of the main chain

* Present address: Department of Physics, Jichi Medical School, 3311-1, Yakushiji, Minami-kawachi, Kawachi-gun, Tochigi-Pref, 329-04, Japan. are numbered from left to right. The calculations were made for two types of hinges (models A and B). In model A, rigid $\alpha$-helices on both sides of the hinge start from the two amide

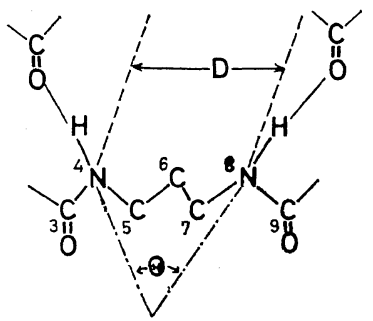

(a)

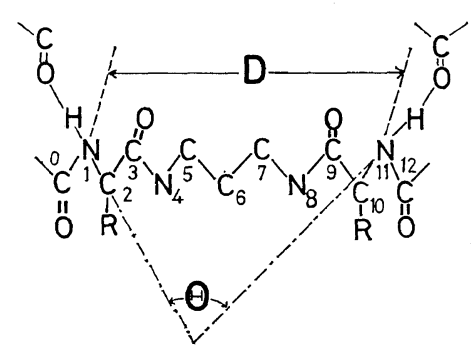

(b)

Figure 1. The diagram which defines $\Theta$ and $D$. $\Theta$ is the angle between two NH-bonds in the peptide units, and $D$ is the distance between two nitrogen atoms in the same peptide units: (a), model A; (b), model $\mathrm{B} ; \mathrm{R},-\mathrm{CH}_{2}-\mathrm{CH}_{2}-\mathrm{CO}-$ $\mathrm{CH}_{2}-\mathrm{C}_{6} \mathrm{H}_{5}$. The numerals represent the order of the atoms in the main chain from left to right. 
groups at positions $(3,4)$ and $(8,9)$, respectively, by making hydrogen bonds with the carbonyl groups three residues ahead. Four internal rotations between atoms 4 and 8 are considered to be released in this model (Figure 1a). In model $B$, on the other hand, the hydrogen atoms in the amide residues at atoms 4 and 8 do not make hydrogen bonds, and rigid $\alpha$-helices start from two amide groups at positions $(0,1)$ and $(11,12)$. Ten internal rotations between atoms 1 and 11 are considered to be released in this model (Figure 1b).

Calculations were made to determine the potential barrier of internal torsional energy of the hinge as a function of the distance, $D$, between nitrogen atoms (4-8 in the model $\mathrm{A}$ and 1-11 in the model B) in two peptide units, and the angle, $\Theta$, between $\mathrm{N}-\mathrm{H}$ bonds in the same peptide units. $\Theta$ can be calculated by

$$
\Theta=\cos ^{-1}\left(\frac{\left(l_{4}^{(4)} \cdot \boldsymbol{l}_{8}^{(4)}\right)}{l_{\mathrm{NH}}^{2}}\right)
$$

for model $\mathrm{A}$, and

$$
\Theta=\cos ^{-1}\left(\frac{\left(l_{1}^{(1)} \cdot l_{11}^{(1)}\right)}{l_{\mathrm{NH}}^{2}}\right)
$$

for model $\mathrm{B}$, where $l_{\mathrm{NH}}$ is the bond length of the $\mathrm{N}-\mathrm{H}$ bond, and $\boldsymbol{l}_{i}^{(j)}$ denotes the $\mathrm{N}-\mathrm{H}$-bond vector at the position $i$, defined in the $j$-th reference frame. Since

$$
\boldsymbol{l}_{8}^{(4)}=\mathbf{T}_{4} \mathbf{T}_{5} \mathbf{T}_{6} \mathbf{T}_{7} \boldsymbol{l}_{8}^{(8)}
$$

and

$$
l_{11}^{(1)}=\mathbf{T}_{1} \mathbf{T}_{2} \ldots \mathbf{T}_{9} \mathbf{T}_{10} \boldsymbol{l}_{11}^{(11)}
$$

by using an orthogonal matrix $\mathbf{T}_{i}$ defined later,

$$
\Theta=\cos ^{-1}\left(\frac{\boldsymbol{l}_{4}^{(4)} \cdot \mathbf{T}_{4} \mathbf{T}_{5} \mathbf{T}_{6} \mathbf{T}_{7} \boldsymbol{l}_{8}^{(8)}}{l_{\mathrm{NH}}^{2}}\right)
$$

for model A, and

$$
\Theta=\cos ^{-1}\left(\frac{l_{1}^{(1)} \cdot \mathbf{T}_{1} \mathbf{T}_{2} \ldots \mathbf{T}_{9} \mathbf{T}_{10} l_{11}^{(11)}}{l_{\mathrm{NH}}^{2}}\right)
$$

for model B. Since $D$ has been defined to be the distance from the nitrogen atom at one end of the hinge to that at the other end,

$$
D=\boldsymbol{l}_{5}+\mathbf{T}_{5}\left(\boldsymbol{l}_{6}+\mathbf{T}_{6}\left(\boldsymbol{l}_{7}+\mathbf{T}_{7} \boldsymbol{l}_{8}\right)\right)
$$

for model $A$, and

$$
\begin{aligned}
D= & l_{2}+\mathbf{T}_{2}\left(\boldsymbol{l}_{3}+\mathbf{T}_{3}\left(\boldsymbol{l}_{4}+\mathbf{T}_{4}\left(\boldsymbol{l}_{5}+\mathbf{T}_{5}+\left(\boldsymbol{l}_{6}+\mathbf{T}_{6}\right.\right.\right.\right. \\
& \left.\left.\left.\left.+\left(\boldsymbol{l}_{7}+\mathbf{T}_{7}\left(\boldsymbol{l}_{8}+\mathbf{T}_{8}\left(\boldsymbol{l}_{9}+\mathbf{T}_{9}\left(\boldsymbol{l}_{10}+\mathbf{T}_{10} \boldsymbol{l}_{11}\right)\right)\right)\right)\right)\right)\right)\right)
\end{aligned}
$$

for model B. Here $l_{i}$ is the bond vector from $(i-1)$-th atom to $i$-th atom defined in the $i$-th reference frame, and $i$ is the atom number defined in Figure 1. The matrix $\mathbf{T}_{i}$ is defined as

$$
\mathbf{T}_{i}=\left[\begin{array}{ccc}
\cos \theta_{i} & \sin \theta_{i} & 0 \\
\sin \theta_{i} \cos \phi_{i} & -\cos \theta_{i} \cos \phi_{i} & \sin \theta_{i} \\
\sin \theta_{i} \sin \phi_{i} & -\cos \theta_{i} \sin \phi_{i} & -\cos \phi_{i}
\end{array}\right]
$$

where $\theta_{i}$ is the supplement of the $i$-th skeletal bond angle between the $i$-th and $(i+1)$-th bond vectors and $\phi_{i}$ is the dihedral angle between the two planes defined, respectively, by the bond pairs $(i-1, i)$ and $(i, i+1)$. The sign of $\phi$ is taken as positive for a right-handed rotation. It is measured relative to the trans conformation. The diagram for illustrating $l, \theta$, and $\phi$ is shown in Figure 2 following the definition of Flory. $4 \mathrm{a}$

In the numerical calculations, $l$ and $\theta$ 's were fixed at the values listed in Table I, and only $\phi$ 's were taken as variables. Since the N-H bond and the $\mathrm{C}=\mathrm{O}$ bond in the peptide unit are considered to be in trans position, $\phi_{4}$ and $\phi_{9}$ were taken to be zero. As the allowed regions of $\phi_{2}, \phi_{3}, \phi_{5}, \phi_{8}, \phi_{10}$, and $\phi_{11}$ in the configurational space, the areas surrounded by contour lines of $1 \mathrm{kcal}$ have been employed. These were calculated by Ponnuswamy and Sasisekharan ${ }^{5}$ for $n$ butyl residue in dipeptide units, as shown in Figure 3. The $\gamma$-carbon of the $n$-butyl residue takes three conformations whose energy contour lines are different. To investigate the allowed region in the configurational space of the hinge, however, it may not make much difference which conformation the $\gamma$-carbon of the residue takes. Therefore, instead of the three contour maps, which are shown by three kinds of broken lines

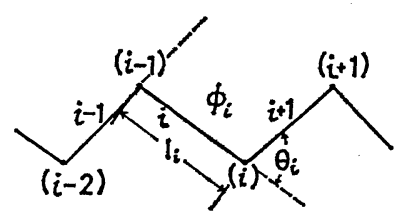

Figure 2. The diagram which represents $l, \theta$ and $\phi$. The definitions are the same as those by Flory. ${ }^{4}$ 
Table I. Bond lengths $l$ and bond angles $\theta$ used in the calculation ${ }^{12}$
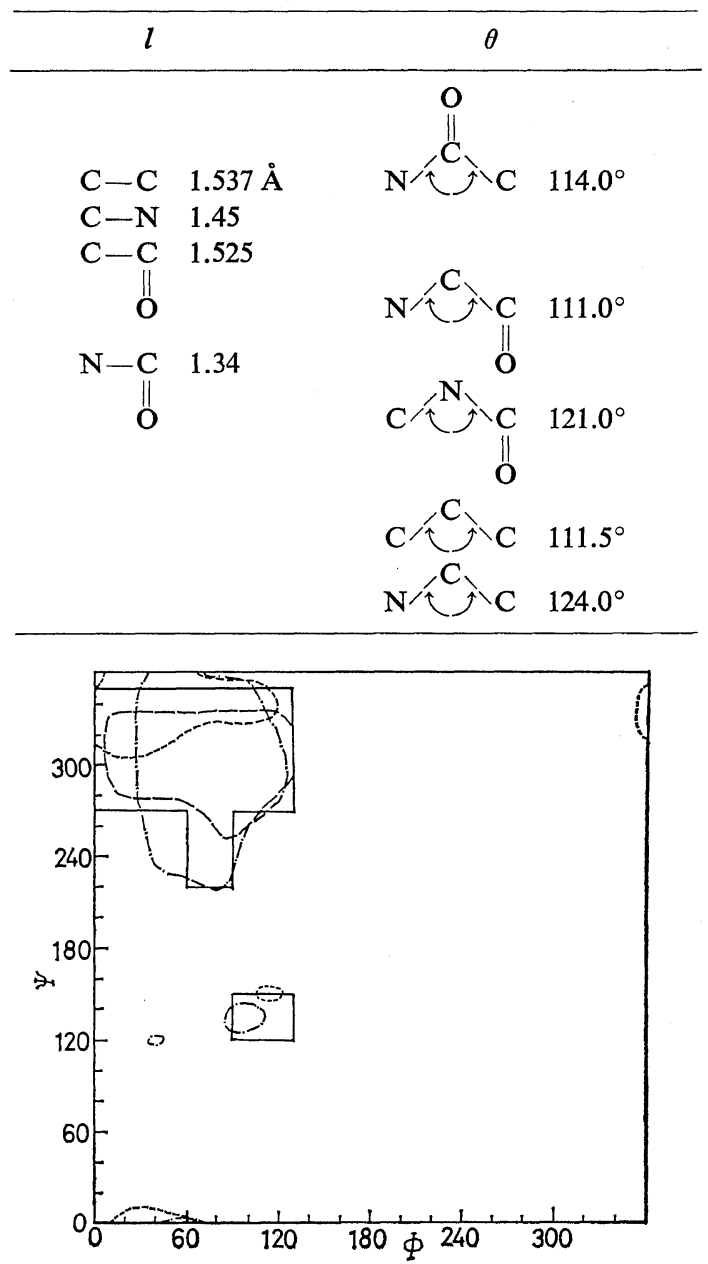

Figure 3. The energy contour map of the $n$-butyl residue in dipeptides; $\phi$ and $\Psi$, rotational angles. ${ }^{5}$ The three broken lines are $1 \mathrm{kcal}$ lines corresponding to three local minimal cases of $\gamma$-carbon. The contour line drawn by the solid line is employed in the numerical calculation for simplicity.

superposed in Figure 3, the contours given by solid lines in the same figure were used for the calculation. As the allowed region of $\phi_{6}$ and $\phi_{7}$ in the configurational space, the areas surrounded by contour lines of $500 \mathrm{cal}$ and $1 \mathrm{kcal}$ have been employed. These were made by Flory ${ }^{4 b}$ in the case of $n$-butane (Figure 4). In the calculation, the elliptic contours have been approximated by squares for the sake of simplicity,

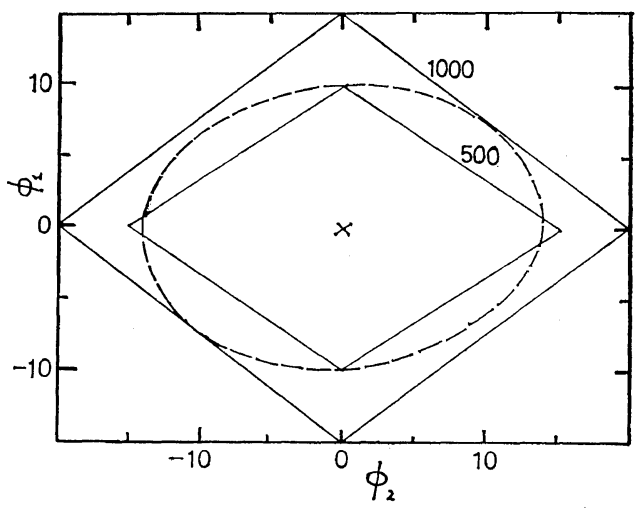

(a)

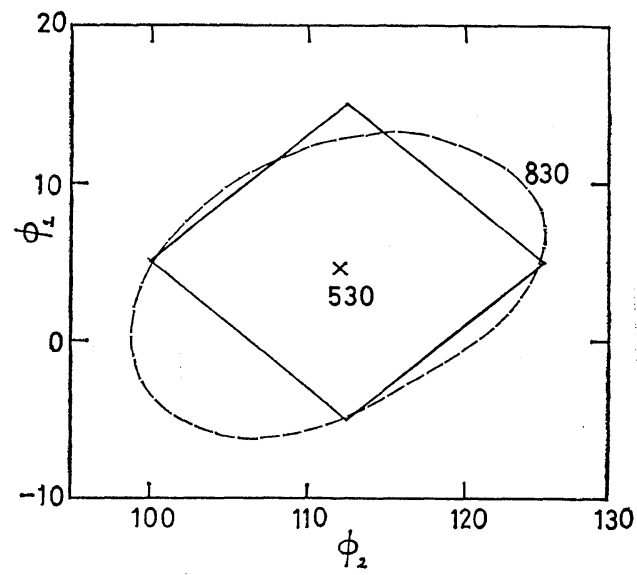

(b)

Figure 4. The energy contour map of $n$-butane: $\phi_{1}$ and $\phi_{2}$, internal rotational angles defined by Flory; 4 broken lines, $500 \mathrm{cal}$ higher than the local minimal energy: (a), trans conformation; (b), gauche conformation. The local minimal energy in this case is $530 \mathrm{cal}$ higher than in the trans conformation. The solid lines are employed as contour lines of $500 \mathrm{cal}$ and $1 \mathrm{kcal}$ for the sake of simplicity.

as shown in the figure.

The energy contour maps calculated are shown in Figures 5 and 6. They indicate that $-\mathrm{NH}-\underset{l}{\mathrm{CH}}-\mathrm{CO}-\mathrm{NH}-\mathrm{CH}_{2}-\mathrm{CH}_{2}-\mathrm{CH}_{2}-$ $-\mathrm{NH}-\mathrm{CO}-\mathrm{CH}-\mathrm{NH}-($ model $\mathrm{B})$ may be flex${ }_{\mathbf{R}}^{1}$

ible enough for the hinge to move with a tor- 


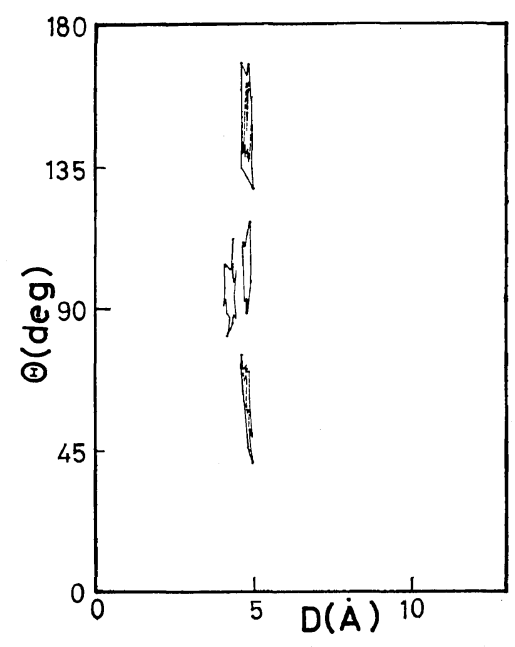

Figure 5. The energy map of model A: broken lines, $500 \mathrm{cal}$; solid lines, $1 \mathrm{kcal}$ higher than the minimal energy.

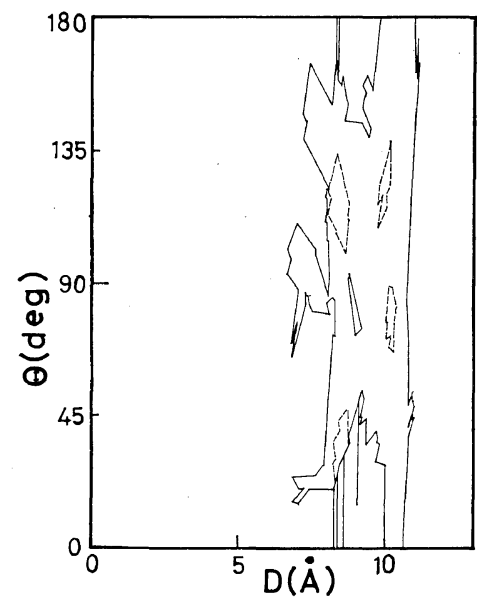

Figure 6. The energy map of model B: broken lines, $500 \mathrm{cal}$; solid lines, $1 \mathrm{kcal}$ higher than the minimal energy.

sional potential barrier of less than $1 \mathrm{kcal}$, but $-\mathrm{NH}-\mathrm{CH}_{2}-\mathrm{CH}_{2}-\mathrm{CH}_{2}-\mathrm{NH}-(\operatorname{model} \mathrm{A})$ is not. The hinge of the model $\mathrm{B}$ type can move freely at room temperature.

\section{DISCUSSION}

There are three degrees of freedom $\left(D, \Theta^{\prime}\right.$, and $\omega$ in Figure 8 ) needed to describe the con- formation of the system completely. However, the electrostatic interaction energy between helices, $E_{\mathrm{e} 1}$, depends only slightly on $\omega$, if the helices are much longer than $D . E_{\text {e1 }}$ may be approximated by the interaction energy among two positive electric charges at the tails of the helices and two negative electric charges at the heads

$E_{\mathrm{e} 1}=e^{2}\left\{\frac{1}{\left|\overrightarrow{l_{2}}+\vec{D}-\vec{l}_{1}\right|}+\frac{1}{D}-\frac{1}{\left|\vec{D}+\overrightarrow{l_{2}}\right|}-\frac{1}{\left|\vec{D}-\overrightarrow{l_{1}}\right|}\right\}(5)$

where $\vec{l}_{1}$ and $\vec{l}_{2}$ are the vectors representing two helices whose directions are from head to tail, $\vec{D}$ is the vector representing the hinge, and $e$ is the charge.

Now, for the case that $\left|\overrightarrow{l_{1}}\right| \simeq\left|\vec{l}_{2}\right| \gg D$, eq 5 can be rewritten as

$$
E_{\mathrm{e} 1} \simeq e^{2}\left\{\frac{1}{\left|\vec{l}_{2}-\vec{l}_{1}\right|}+\frac{1}{D}-\frac{1}{l_{1}}-\frac{1}{l_{2}}+0\left(\frac{D}{l_{1}^{2}}\right)\right\}
$$

$\omega$ does not influence the first four terms, and $E_{\mathrm{e} 1}$ depends on $\omega$ only in the order of $D / l_{1}^{2}$. As the interaction energy of the hinge, $E_{\text {hinge }}$, is indifferent to $\omega$, it influences the total interaction energy ( $\left.=E_{\mathrm{e} 1}+E_{\text {hinge }}\right)$ only in the order of $D / l_{1}^{2}$; thus the freedom about $\omega$ need not be considered. The angle between the two helices, $\Theta^{\prime}$, is not equal to the $\Theta$ used in the calculation of the energy contour map. There is, however, a one to one correspondence between them.

Since it has been reported ${ }^{6}$ that the $\delta$-carbon and atoms beyond have little effect on the backbone conformation in dipeptide units, the difference of the residue attached to the $\delta$-carbon may have no effect on the main chain conformation. From the calculation it is found that, if the hydrogen atoms of the amine groups in the initiator take part in the hydrogen bond with the carbonyl group three residues ahead (model A), the hinge is far from a free joint with potential barrier less than $1 \mathrm{kcal}$, the case shown in Figure 5. If this is not the case (model B), the hinge can work as a free joint of low energy barrier less than $1 \mathrm{kcal}$ (Figure 6).

To investigate the long-range interaction, we built a molecular model of hinged poly( $\gamma$-benzylL-glutamate). The model was constructed using the HGS molecular structure model kit (Maru- 


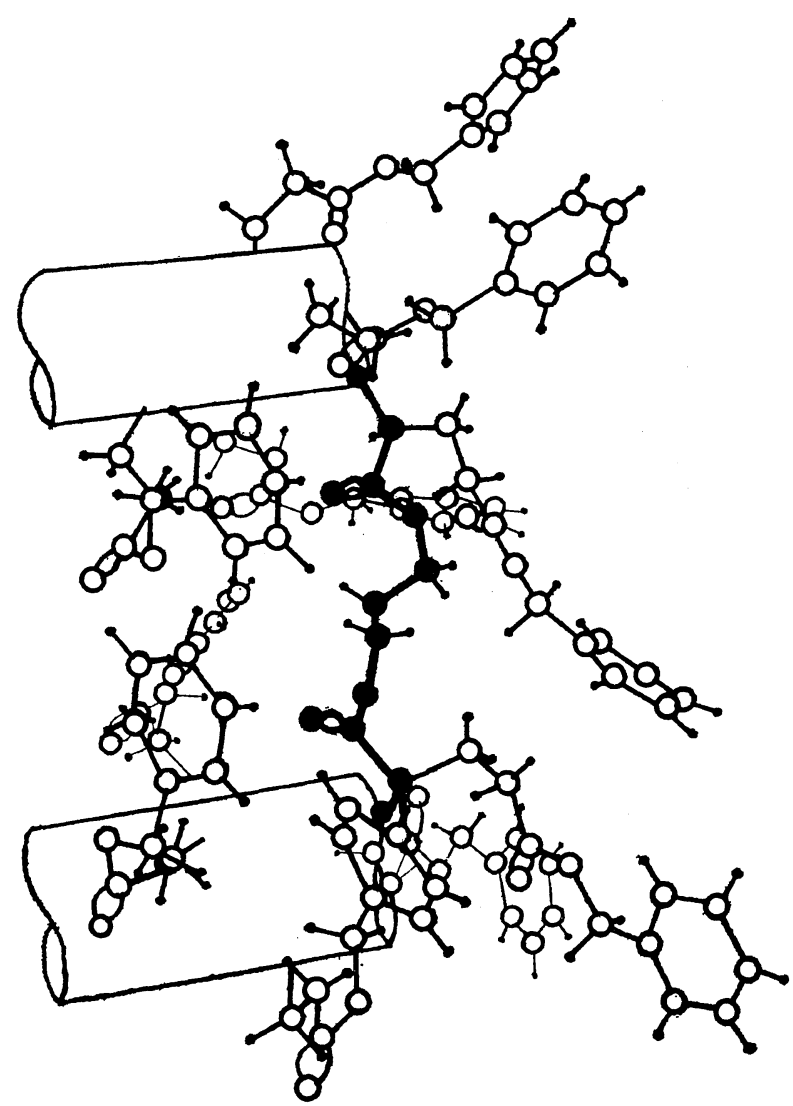

Figure 7. The molecular model of hinged PBLG for $\Theta=0^{\circ}$ with several side chains. The cylinders represent $\alpha$-helices. Black circles and thick bonds represent the hinge of model $B$.

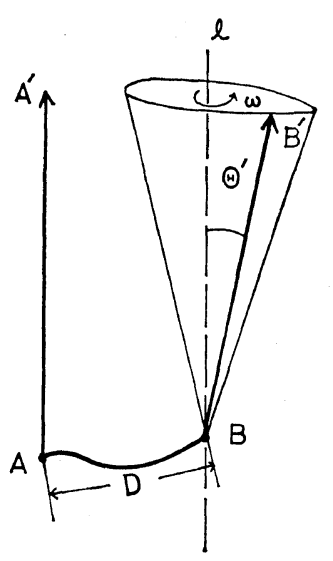

Figure 8. The relations among $\omega, \Theta^{\prime}$, and $\Theta: l$, parallel to $\mathrm{AA}^{\prime} ; \Theta^{\prime}$, the angle between $l$ and $\mathbf{B B}^{\prime}$ corresponding to $\Theta$ on a one to one basis; $\omega$, the angle around $l$. zen, JAPAN). The $\mathrm{N}-\mathrm{H}$ and $\mathrm{C}-\mathrm{O}$ bonds in the peptide units were fixed in trans conformation. ${ }^{11}$ Since the precise conformation of the side chain is not known, $7 \mathrm{~b}, 9,10,13$ the conformation having the largest distance between the benzene ring and the $\alpha$-carbon has been employed. The angle between the benzene ring and the main chain is fixed at $60^{\circ} .7 \mathrm{a}$ Figure 7 shows the configuration when $\theta=0$. There seems to be no steric hindrances to prevent the two $\alpha$-helices from moving in all directions. Incidentally, Teramoto ${ }^{8}$ has indicated that five or six amino acid residues exist as unfolded at the end of a single $\alpha$-helix, and two or so amino acid residues of the $\alpha$-helix from the initiation point are perhaps free from the hydrogen bond in the hinged polymer.

From the dielectric study, the following quan- 


\section{H. Kihara and A. WADA}

tity can be obtained

$$
\int_{\Omega} \cos \Theta \exp \{-F / k T\} \mathrm{d} \Omega
$$

where $F$ and $k T$ are Helmholtz free energy and thermal energy respectively, and the integral is performed over all the configurations. As previously reported, eq 7 has a value nearly equal to zero, which means (a) the hinge is not flexible and the energy minimum of $E_{\text {hinge }}$ exists when $\Theta^{\prime}$ is about $90^{\circ}$ (model $A$ ) or (b) hinge is flexible, and the slight deviation of the value of eq 7 from 0 is accounted for by the electrostatic interaction energy (model B or a longer hinge). Considering Teramoto's above-mentioned suggestion model $\mathrm{B}$ or a longer hinge is more probable.

Finally, for an empirical confirmation of the flexibility of the hinge, polymerization was carried out using hexamethylenediamine as an initiator as well as trimethylenediamine. The results of dielectric dispersion of these two types of molecules were found to be quite similar as far as total dipole moments were concerned. This indicates that a hinge of this length has little effect on the conformation of the total molecule. The details of the data and discussions about this point were reported previously. ${ }^{1}$

From these points, the hinge of the hinged polymer can be concluded to be perfectly or nearly perfectly flexible.

Acknowledgement. The authors wish to thank
Miss Kimie Hamano, who helped to make the energy contour maps.

\section{REFERENCES}

1. H. Kihara and A. Wada, Polymer J., 5, 324 (1973).

2. K. Nakagawa, N. Nishioka, A. Teramoto, and H. Fujita, ibid., 4, 332 (1973).

3. A. Teramoto, T. Yamashita, and H. Fujita, J. Chem. Phys., 46, 1919 (1967).

4. (a) P. J. Flory, "Statistical Mechanics of Chain Molecules," Interscience, New York, N.Y., 1969, p 20; (b) ibid., p 137.

5. P. K. Ponnuswamy and V. Sasisekharan, Biopolymers, 10, 565 (1971).

6. V. Sasisekharan and P. K. Ponnuswamy, ibid., 10, 583 (1971).

7. (a) E. Charney, J. B. Milstien, and K. Yamaoka, J. Amer. Chem. Soc., 92, 2657 (1970); (b) M. Tsuboi, J. Polym. Sci., 59, 139 (1962).

8. A. Teramoto, private communication.

9. J. F. Yan, G. Vanderkooi, and H. A. Scheraga, J. Chem. Phys., 49, 2713 (1968).

10. E. M. Bradbury, B. G. Carpenter, C. CraneRobinson, and H. Goldman, Macromolecules, 4, 557 (1971).

11. R. B. Corey and L. Pauling, Proc. Roy. Soc. (London), Ser. B, 141, 10 (1953).

12. Y. Kawade, E. Teramoto, and A. Wada, Ed., "Seibutsu Butsuri Jikken Handbook" (Japanese), Yoshioka-Shoten, Tokyo, 1970, p. 125.

13. K. Nagayama and A. Wada, Abstracts, 12th Annual Meeting of Biophysics Society of Japan, Tokyo, Oct. 1973, p 212. 